\title{
Améliorer la réglementation du cannabis médical au Canada pour mieux servir les patients pédiatriques
}

\author{
Richard J. Huntsman MD, Lauren E. Kelly PhD, Jane Alcorn DMV PhD, Juan Pablo Appendino MD, \\ Richard E. Bélanger MD, Bruce Crooks MBChB, Yaron Finkelstein MD, Andrea Gilpin PhD MBA, Evan Lewis MD, \\ Catherine Litalien MD, Julia Jacobs MD, Charlotte Moore-Hepburn MD, Timothy Oberlander MD, \\ S. Rod Rassekh MD MScS, Alexander E. Repetski; Michael J. Rieder MD PhD, Alan Shackelford MD, \\ Hal Siden MD MScS, Michael Szafron PhD, Geert W. 't Jong, MD PhD, Régis Vaillancourt PharmD, pour la Cannabinoid \\ Research Initiative of Saskatchewan et le Consortium C4T (Canadian Childhood Cannabinoid Clinical Trial).
}

Citation : CMAJ 2021 October 18;193:E1596-9. doi : 10.1503/cmaj.202169-f

Voir la version anglaise de l'article ici : www.cmaj.ca/lookup/doi/10.1503/cmaj.202169

$\mathbf{L}$ es enfants atteints de maladies chroniques invalidantes et douloureuses utilisent de plus en plus le cannabis médical, particulièrement lorsque les options thérapeutiques classiques sont peu efficaces ou provoquent des effets indésirables substantiels. De plus en plus, les aidants sont au courant de travaux démontrant que le cannabis médical offre des bienfaits aux enfants qui présentent des affections telles que l'épilepsie pharmacorésistante, la douleur cancéreuse et l'autisme $^{1,2}$. À l'heure actuelle, l'accès au cannabis pour les enfants et les jeunes de 18 ans et moins (ci-après, les enfants) est encadré par Santé Canada. En 2018, la Loi sur le cannabis (loi C-45) a prévu des volets distincts pour l'achat de cannabis à des fins médicales et à des fins récréatives ${ }^{3,4}$. En vertu de cette loi, un traitement pédiatrique à base de cannabis doit être autorisé par un médecin ou une infirmière praticienne, ce qui donne l'occasion de faire de l'enseignement au patient (ou à ses aidants). Des règles strictes ont été instaurées pour la production et l'étiquetage afin de limiter les contaminants et d'assurer une certaine uniformité dans la teneur en cannabinoïdes, ce qui est une bonne chose pour les enfants qui utilisent du cannabis ${ }^{5}$. Toutefois, les règles encadrant l'autorisation médicale sont dépassées et ne répondent pas aux besoins de cette population, et l'étiquetage des produits demeure inadéquat, tout comme la surveillance des réactions indésirables chez les enfants. Il faut donc améliorer la réglementation.

En vertu des règles actuelles, les professionnels de la santé doivent préciser la quantité de cannabis autorisée pour leurs patients en grammes de cannabis séché par jour. Durant la deuxième moitié de 2018, les extraits de cannabis (préparations

\section{Points clés}

- Les enfants atteints de maladies chroniques invalidantes et douloureuses utilisent de plus en plus le cannabis médical, particulièrement lorsque les options thérapeutiques classiques se révèlent peu efficaces ou produisent des effets indésirables substantiels.

- Il faut améliorer les processus et la réglementation au Canada afin que des recherches adéquates sur l'efficacité et les effets indésirables des produits de cannabis utilisés à des fins médicales puissent être réalisées chez les enfants et les adolescents.

- Une amélioration des processus d'autorisation et de prescription et l'obligation de déclarer les effets indésirables s'imposent si l'on veut améliorer la sécurité des patients.

- Des mécanismes novateurs pour le financement adéquat de la recherche indépendant de l'industrie sont nécessaires afin d'appuyer la recherche et de renforcer les bases de données probantes sur les indications, la posologie, l'efficacité et la toxicité potentielle du cannabis médical chez les patients pédiatriques.

obtenues à partir des feuilles et des fleurs [inflorescence] de Cannabis sativa) représentaient $67 \%$ du cannabis autorisé à des fins médicales au Canada ${ }^{6}$. Même si nous n'avons pas actuellement accès à des données pédiatriques, l'expérience clinique indique que ce sont surtout les extraits, et non les produits séchés, qui sont surtout utilisés chez les enfants. Si les patients utilisent des extraits, les fournisseurs de soins doivent en convertir la posologie pour remplir le formulaire d'autorisation. La teneur en cannabinoïdes du cannabis séché utilisé pour préparer 
les extraits varie, ce qui complique la conversion de la posologie quotidienne souhaitée de milligrammes de cannabinoïdes en grammes de cannabis séché. En outre, différents procédés d'extraction des cannabinoïdes à partir du cannabis séché risquent de donner à l'extrait qui en résulte un profil distinct de cannabinoïdes, de terpénoïdes et de solvants résiduels ${ }^{7}$. Par ailleurs, il peut aussi y avoir une variabilité d'un lot à l'autre d'un même produit chez un producteur de cannabis donné. Et le calcul des doses se complique encore davantage si le patient utilise plus d'une préparation.

Bien que les producteurs autorisés doivent utiliser des méthodes validées pour mesurer les concentrations de cannabinoïdes, Santé Canada requiert seulement qu'ils inscrivent sur l'étiquette les concentrations totales de cannabidiol (CBD) et de tétrahydrocannabinol (THC), combinant les formes carboxylées et décarboxylées (activées) ${ }^{5}$. La variabilité de la teneur en CBD, en THC et en autres cannabinoïdes et terpènes non divulgués complique la détermination du profil d'innocuité et d'efficacité, décourage les professionnels de la santé de prescrire du cannabis et empêche l'inscription des extraits de cannabis sur les listes de médicaments des provinces, des territoires et de nombreux régimes d'assurance médicaments privés ${ }^{8}$. Par conséquent, les extraits de cannabis médical sont inabordables pour beaucoup d'aidants d'enfants, ce qui les force à se tourner vers le cannabis récréatif ou à produire leurs propres extraits maison plus ou moins sécuritaires ${ }^{8}$.

Au Canada, la recherche sur l'utilisation du cannabis médical chez les enfants est freinée par un manque de données nationales adéquates. Santé Canada tient des statistiques sur l'utilisation du cannabis médical, mais ne les stratifie actuellement pas selon l'âge. Santé Canada maintient en outre un système de déclaration des effets indésirables associés aux produits dérivés du cannabis sur le site Web du Programme Canada Vigilance ${ }^{9}$. Les producteurs de cannabis autorisés sont tenus de déclarer tout effet indésirable grave associé à l'utilisation de leurs produits, mais les professionnels de la santé n'y sont pas contraints. Étant donné que les patients sont susceptibles de signaler les effets indésirables à leur professionnel de la santé ou à consulter celui-ci lorsqu'un effet grave survient, certains incidents pourraient ne pas être déclarés à Santé Canada. Des données nationales sur les indications et les effets indésirables de même que sur les différents types et les doses de produits de cannabis médical utilisés chez les enfants seraient d'une aide précieuse.

La recherche sur l'efficacité est également freinée par la réglementation actuelle sur la production du cannabis; cela a pour effet que l'utilisation des produits de cannabis médical chez les enfants augmente rapidement malgré l'absence de connaissances fiables issues d'essais cliniques. La production, l'étiquetage et la distribution du cannabis médical et récréatif suivent le Guide des bonnes pratiques de production (BPP) du cannabis de Santé Canada, ce qui inspire une certaine confiance en leur qualité ${ }^{5}$. En revanche, les Lignes directrices sur les bonnes pratiques de fabrication (BPF) de Santé Canada pour les produits pharmaceutiques et de santé naturelle exigeraient une documentation plus stricte et d'autres tests pour quantifier les concentrations de $\mathrm{THC}$ et de $\mathrm{CBD}$. De récentes modifications apportées par Santé Canada exigent un certificat de conformité aux BPF et une brochure de l'investigateur pour que les produits de cannabis puissent être utilisés dans des essais cliniques ${ }^{10}$. La plupart des producteurs de cannabis autorisés n'ont ni les ressources ni l'expertise pour procéder à la recherche préclinique qui permettrait de produire une brochure de l'investigateur pour leurs produits, ce qui limite substantiellement le nombre de produits utilisables pour des essais cliniques. Par ailleurs, le coût élevé des essais cliniques combiné à un manque de financement indépendant de l'industrie nuit à la réalisation de recherches solides. Pour combler le manque de financement, des partenariats de recherche sont formés entre certains producteurs de cannabis autorisés et des établissements universitaires canadiens, mais cela soulève des inquiétudes quant à l'indépendance de la recherche.

En plus de l'absence de travaux de recherche de grande qualité, et plus spécifiquement, de l'absence de données probantes au sujet des effets neurodéveloppementaux à long terme potentiels du cannabis chez les enfants, la formation lacunaire des professionnels de la santé sur les indications, l'efficacité, la posologie et l'innocuité des produits du cannabis à usage médical accentue leur réticence à en autoriser l'utilisation chez les enfants $^{11}$. En outre, la disparité des règlements d'une province et d'un territoire à l'autre ajoute au questionnement des professionnels de la santé quant au bien-fondé de la prescription du cannabis médical chez les enfants.

Les processus et règlements actuels pourraient être améliorés de plusieurs façons pour soutenir l'utilisation sécuritaire des cannabinoïdes chez les enfants.

Les formulaires d'autorisation des médecins pour le cannabis médical devraient permettre que la dose quotidienne soit exprimée en milligrammes de cannabinoïdes par jour, et que le rapport désiré de cannabinoïdes soit exprimé en milligrammes par millilitre (p. ex., 1:20 THC:CBD). Pour garantir que les patients ne subissent pas d'interruption de leur approvisionnement en cannabis médical, un éventail de concentrations pourrait être proposé à l'intérieur du rapport désiré (p. ex., 0,5-1:18-22 THC:CBD). En outre, les formulaires d'autorisation devraient être modifiés afin qu'on puisse servir plus d'un produit à un même patient.

Les producteurs de cannabis autorisés devraient être obligés d'afficher clairement sur les étiquettes de produits les concentrations (en $\mathrm{mg} / \mathrm{mL}$ ) de THC, puis du cannabinoïde principal suivant. Les étiquettes devraient aussi inclure (en plus petits caractères) les concentrations des formes carboxylées et décarboxylées des cannabinoïdes principaux et des autres cannabinoïdes dont la concentration dépasse $1 \mathrm{mg} / \mathrm{mL}$. Cette uniformité dans l'étiquetage pourrait rassurer les aidants et les médecins quant aux potentiels effets psychoactifs et développementaux à long terme du THC chez les enfants.

Bien qu'il ne soit pas réaliste de demander aux producteurs autorisés d'utiliser une seule et même méthode d'extraction normalisée, on pourrait exiger qu'ils indiquent la méthode d'extraction utilisée pour un produit donné, de même que la source du cannabis lui-même. Plusieurs des principaux producteurs autorisés ont déjà pris l'initiative de préparer des 
produits en fonction des normes de BPF, et cela devrait être exigé pour tous les produits de cannabis médical vendus au Canada. Le passage de la certification de conformité aux BPP à celle des BPF pourrait représenter des coûts pour les fournisseurs et entraîner de ce fait une hausse du prix des produits de cannabis médical, mais cela faciliterait la recherche ${ }^{12}$. Exiger une certification de conformité aux BPF pour les produits de cannabis médical pourrait toutefois donner lieu à un système de réglementation à 2 vitesses (avec certificat de conformité aux BPP et aux BPF requis respectivement pour les produits de cannabis à usage récréatif et à usage médical), et certains producteurs pourraient choisir de se retirer du marché du cannabis médical. Pour assurer un approvisionnement adéquat de produits certifiés conformes aux BPF et soutenir adéquatement les essais cliniques pédiatriques, nous suggérons que des mesures incitatives gouvernementales soient offertes (p. ex., exclusivité des brevets et des marchés, comme c'est le cas pour l'industrie pharmaceutique traditionnelle).

Les pharmacies communautaires pourraient être autorisées à servir et à préparer des produits de cannabis médical, puisqu'en majorité, elles disposent déjà de l'infrastructure et des chaînes d'approvisionnement nécessaires pour commander et stocker des substances réglementées. Étant donné que les pharmaciens sont formés pour évaluer les interactions médicamenteuses et les effets indésirables, nous appuyons l'énoncé de position de l'Association des pharmaciens du Canada sur le cannabis médical, selon lequel ces professionnels sont les mieux placés pour conseiller les patients (ou leurs aidants), superviser le stockage sécuritaire du cannabis et servir les produits ${ }^{13}$.

Santé Canada devrait stratifier les données sur l'utilisation du cannabis médical selon l'âge et le type de préparation, et demander que soient déclarés tous les effets indésirables graves associés au cannabis médical, y compris par les pharmaciens. Ces déclarations pourraient se faire par l'entremise du Programme Canada Vigilance, en conjonction avec le Programme canadien de surveillance pédiatrique existant. Tout système de déclaration devrait être facilement accessible (c.-à-d., sur ordinateur ou appareil intelligent), et rapide et simple à utiliser.

Enfin, il faudra mettre sur pied un mécanisme de financement adéquat de la recherche qui soit indépendant de l'industrie. Une infrastructure de recherche nationale efficace existe déjà avec des organisations telles que le Consortium C4T (Canadian Childhood Cannabinoid Clinical Trials, www.C4Trials.org), le Canadian Consortium for the Investigations of Cannabinoids et la Cannabinoid Research Initiative of Saskatchewan. Ces groupes ont tout ce qu'il faut pour procéder à des recherches pédiatriques multicentriques de grande qualité sur le cannabis médical au Canada. Si le financement adéquat était disponible, le Canada pourrait être en bonne position pour contribuer grandement aux connaissances sur l'utilisation sécuritaire et efficace du cannabis médical chez les enfants dont les besoins thérapeutiques ne sont pas adéquatement comblés. Les partenariats public-privé peuvent jouer un rôle important dans le financement de la recherche. Par exemple, un partenariat entre la Fondation de l'Hôpital pour enfants Jim Pattison et la Fondation de l'Hôpital pour enfants du Manitoba, et la Fondation pour la recherche en santé de la Saskatchewan et
Recherche Manitoba est en cours d'élaboration pour créer un mécanisme par lequel les producteurs autorisés pourront verser des fonds non dirigés pour la recherche sur le cannabis chez les enfants. Une fois les partenariats établis, les chercheurs pourront demander du financement par l'entremise d'un processus de révision par des pairs de la Fondation pour la recherche en santé de la Saskatchewan et de Recherche Manitoba. Une coordination intergouvernementale visant l'application à la recherche d'un pourcentage des taxes perçues sur les ventes de cannabis à usage récréatif (notamment sur l'utilisation du cannabis chez les enfants) favoriserait également la création d'une meilleure base de connaissances.

\section{Références}

1. Pawliuk C, Chau B, Rassekh SR, et al. Efficacy and safety of paediatric medicinal cannabis use: A scoping review. Paediatr Child Health 2020;26:228-33.

2. Aran A, Cassuto H, Lubotzky A, et al. Brief report: cannabidiol-rich cannabis in children with autism spectrum disorder and severe behavioral problems - a retrospective feasibility study. J Autism Dev Disord 2019;49:1284-8.

3. An Act respecting cannabis and to amend the Controlled Drugs and Substances Act, the Criminal Code and other acts. 42nd Parliament, 1st sess, 2018 June 21. Accessible ici : https://www.parl.ca/DocumentViewer/en/42-1/bill/c-45/royal-assent (consulté le 25 sept. 2021).

4. Cannabis for medical purposes under the Cannabis Act: information and improvements. Ottawa: Health Canada; modified 2021 May 19. Accessible ici : https://www.canada.ca/en/health-canada/services/drugs-medication/cannabis /medical-use-cannabis.html (consulté le 25 sept. 2021).

5. Guidance document: Good production practices guide for cannabis. Ottawa: Health Canada; modified 2019 Dec. 4. Accessible ici : https://www.canada.ca/en/ health-canada/services/cannabis-regulations-licensed-producers/good-production -practices-guide/guidance-document.html (consulté le 25 sept. 2021).

6. Market data under the Access to Cannabis for medical Purposes Regulations. Ottawa: Health Canada; modified 2019 Aug. 29. Accessible ici : https://www.canada. ca/en/health-canada/services/drugs-medication/cannabis/licensed-producers /market-data.html (consulté le 25 sept. 2021).

7. Hazekamp A. The trouble with CBD oil. Med Cannabis Cannabinoids 2018;1:65-72.

8. Elliott J, DeJean D, Potter BK, et al. Barriers in accessing medical cannabis for children with drug-resistant epilepsy in Canada: a qualitative study. Epilepsy Behav 2020;111:107120.

9. Cannabis recalls, adverse reactions and reporting: report side effects from cannabis products. Ottawa: Health Canada; modified 2020 Dec. 14. Accessible ici : https://www.canada.ca/en/health-canada/services/drugs-medication/cannabis /recalls-adverse-reactions-reporting/report-side-effects-cannabis-products. html (consulté le 25 sept. 2021).

10. Notice to stake holders: clarification of requirements under the Food and Drug Regulations when conducting clinical research with cannabis. Ottawa: Health Canada; modified 2021 May 31. Accessible ici : https://www.canada.ca/en/ health-canada/services/drugs-health-products/drug-products/announcements/ notice-clarification-requirements-conducting-clinical-research-cannabis.html (consulté le 25 sept. 2021).

11. Elliott J, DeJean D, Potter BK, et al. Neurologist's perspectives on medical cannabis for pediatric drug-resistant epilepsy in Canada: a qualitative interview study. Seizure 2020;78:118-26.

12. Mutual recognition agreement between Canada and the European Union. Ottawa: health Canada; modified 2021 May 31. Accessible ici : https://www. canada.ca/en/health-canada/services/drugs-health-products/compliance -enforcement/international/mutual-recognition-agreements/updates/mutual -recognition-agreement-canada-european-community.html (consulté le 25 sept. 2021)

13. Medical cannabis. Ottawa: Canadian Pharmacists Association. Accessible ici : https://www.pharmacists.ca/advocacy/medical-cannabis/ (consulté le 25 sept. 2021). 
Intérêts concurrents : Richard Huntsman et Lauren Kelly sont coprésidents du Comité consultatif scientifique sur les produits de santé contenant du cannabis de Santé Canada; l'opinion du Comité n'est ni reflétée ni garantie dans cet article. Lauren Kelly détient une bourse Mitacs Accélération en partenariat avec Canopy Growth, sans lien avec le présent manuscrit. Juan Pablo Appendino déclare avoir reçu des honoraires de Pendopharm, Sunovion et UCB; il est également membre de comités ou de conseils de la Canadian League Against Epilepsy, de la Société canadienne de neurophysiologie clinique, de la Fédération des sciences neurologiques du Canada et de l'Académie ibéro-américaine de neurologie pédiatrique. Richard Bélanger fait partie du comité sur le cannabis de la Société canadienne de pédiatrie. Bruce Crooks indique avoir participé au comité de surveillance de la sécurité des données pour l'étude ReRAD et il est membre du comité consultatif pour la Nouvelle-Écosse et du comité consultatif médical de la fondation Rêves d'enfants Canada. Evan Lewis est conseiller médical bénévole auprès de JMCC Canada Corp. et déclare avoir reçu des honoraires de Spectrum Therapeutics. Alexander Repetski a occupé un poste de consultant bénévole auprès de Strainprint. Michael Rieder est membre du comité de surveillance de l'innocuité des médicaments pour une étude sur l'utilisation du cannabis médical pour traiter la céphalée chez l'enfant, et il est l'auteur principal du document de principes de la Société canadienne de pédiatrie sur le cannabis médical chez les enfants. Régis Vaillancourt déclare avoir reçu des honoraires de consultation de Harvest Medicine. Aucun financement n'a été versé pour la rédaction de ce manuscrit. Aucun autre intérêt n'a été déclaré.

Cet article a été révisé par des pairs.

Affiliations : Division de neurologie pédiatrique (Huntsman), Département de pédiatrie et Cannabinoid Research Initiative of Saskatchewan (Huntsman, Alcorn, Szafron, Shackelford), Université de la Saskatchewan, Saskatoon, Sask.; Consortium C4T (Canadian
Childhood Cannabinoid Clinical Trial) (Kelly, Crooks, Finkelstein, Gilpin, Oberlander, Rassekh, Repetski, Rieder, Vaillancourt); Centre George et Fay Yee pour l'innovation en soins de santé (Kelly), Institut de recherche de l'Hôpital pour enfants du Manitoba, Département de pédiatrie et de santé infantile, Université du Manitoba, Winnipeg, Man.; École de pharmacie et de nutrition (Alcorn) de l'Université de la Saskatchewan, Saskatoon, Sask.; Division de neurologie pédiatrique (Appendino, Jacobs), Département de pédiatrie et Institut de recherche de l'Hôpital pour enfants de l'Alberta (Appendino, Jacobs), Université de Calgary, Calgary, Alb.; Département de pédiatrie (Bélanger), Faculté de médecine, Université Laval, Québec, Qc; Société canadienne de pédiatrie (Bélanger, Moore-Hepburn, 't Jong), Ottawa, Ont.; Division d'hémato-oncologie pédiatrique (Crooks), Département de pédiatrie, Université Dalhousie, Halifax, N.-É.; Divisions de médecine d'urgence et de pharmacologie et toxicologie cliniques (Finkelstein), Département de pédiatrie, Université de Toronto, Toronto, Ont.; Centre de formulations pédiatriques Goodman du Centre hospitalier universitaire Sainte-Justine (Gilpin, Litalien), Montréal, Qc; Centre de neurologie de Toronto (Lewis); Division de neurologie pédiatrique (Lewis), Département de pédiatrie, Université de Toronto, Toronto, Ont.; Département de pédiatrie (Litalien), Faculté de médecine, Université de Montréal, Montréal, Qc; Division de médecine pédiatrique (Moore-Hepburn), Département de pédiatrie, Université de Toronto, Toronto, Ont.; Département de pédiatrie et École de santé des populations et de santé publique (Oberlander) et Division d'hémato-oncologie et de greffes de moelle osseuse pédiatriques (Rassekh), Département de pédiatrie, Université de la Colombie-Britannique, Vancouver, C.-B.; Division de pharmacologie pédiatrique (Rieder), Département de pédiatrie, Université Western Ontario, London, Ont.; Amarimed of Colorado (Shackelford), Denver (Colorado); Division des soins palliatifs
(Siden), Département de pédiatrie, Université de la Colombie-Britannique; Canuck Place (Siden), Children's Hospice, Vancouver, C.-B.; École de santé publique (Szafron), Université de la Saskatchewan, Saskatoon, Sask.; Départements de pédiatrie et de pharmacologie ('t Jong), Faculté de médecine Max Rady, Université du Manitoba, Winnipeg, Man.; Hôpital pédiatrique de l'Est de l'Ontario (Vaillancourt), Ottawa, Ont.

Collaborateurs : Tous les auteurs ont contribué à la conception du travail, ont rédigé le manuscrit et en ont révisé de façon critique le contenu intellectuel important; ils ont donné leur approbation finale pour la version destinée à être publiée et assument l'entière responsabilité de tous les aspects du travail.

Propriété intellectuelle du contenu : Il s'agit d'un article en libre accès distribué conformément aux modalités de la licence Creative Commons Attribution (CC BY-NC-ND 4.0), qui permet l'utilisation, la diffusion et la reproduction dans tout médium à la condition que la publication originale soit adéquatement citée, que l'utilisation se fasse à des fins non commerciales (c.-à-d., recherche ou éducation) et qu'aucune modification ni adaptation n'y soit apportée. Voir : https://creativecommons.org/ licenses/by-nc-nd/4.0/deed.fr.

Remerciements : Les auteurs tiennent à remercier les membres suivants de la Cannabinoid Research Initiative of Saskatchewan et du Consortium C4T (Canadian Childhood Cannabinoid Clinical Trial) pour leur contribution et leur expertise lors de la révision du manuscrit : les $D^{\text {rs }}$ Blair Seifert et Declan Quinn, de même que Simona Meier et Scott Corley. Ils souhaitent aussi exprimer leur gratitude à l'endroit du Maternal Infant Child and Youth Research Network et de son directeur scientifique, le $D^{r}$ Thierry Lacaze-Masmonteil, pour leur soutien constant au Consortium C4T. Leur aide a permis la mise en place d'un cadre national de collaboration entre les auteurs.

Correspondance : Richard Huntsman, dr.huntsman@usask.ca 